\title{
Combined Optical Raytrace and Monte-Carlo Simulation of Complex Biological Imaging
}

\author{
Guillem Carles and Andrew R. Harvey \\ University of Glasgow, University Avenue, Glasgow, G12 8QQ, UK \\ andy.harvey@glasgow.ac.uk
}

\begin{abstract}
We report the first holistic modelling of optical systems for imaging in turbid media. Using a user-friendly commercial optical-design programme, we report hybrid raytracing and Monte-Carlo propagation to optimise optical fluorescence microscope systems and ophthalmoscopes. (C) 2019 The Author(s)
\end{abstract}

OCIS codes: ((170.3880) Medical and biological imaging; (110.0113) Imaging through turbid media; (290.4210) Multiple scattering.

\section{Introduction}

Techniques such as light-sheet microscopy, confocal microscopy, photoacoustics or optical coherence tomography, have been developed to mitigate the light scattering, which prevents optical imaging deep in tissue [1]. Development and understanding such techniques requires accurate modelling of light propagation in optical instruments and in turbid media. Monte-Carlo modelling is the gold standard for simulating photon transport in tissue, and optical raytracing is the established method for modelling light propagation through optical components $[1,2]$. In principle both are based on equivalent fundamental principles: however ray tracing is deterministic, while Monte Carlo is stochastic. Their integration provides a powerful tool for the holistic simulation of imaging systems including arbitrarily complex biological media and structures. Importantly, this integration enables the rigorous tracking of polarisation and coherence of light from source to detector.

We report here simulation examples and provide a set of add-on supplementary software packages to ZemaxOpticStudio ${ }^{\circledR}[3,4]$ to enable for the first time such holistic modelling through a graphical interface.

\section{Simulation examples}

We report two simulation examples. First, the modelling of retinal imaging with a Scanning Laser Ophthalmoscope, Fig. 1, where an illumination spot at the retina is scanned whilst recording the backscattered light that exits the eye. A schematic model of a human eye is used that includes 3D vasculature networks embedded into the retina. Light is absorbed by haemoglobin in blood vessels, resulting in the contrasted retinal images shown in $(b, c)$. Image contrast is the result of multiple light paths from stochastic scattering. Because the extinction coefficient of haemoglobin has a dependency on oxygen saturation at some wavelengths but reduced or no dependency at other wavelengths, it is possible to infer oxygen saturation by multi-spectral imaging. Results for so-called two-wavelength oximetry are shown in (d), employing laser illumination at $532 \mathrm{~nm}$ and $633 \mathrm{~nm}$ wavelengths.
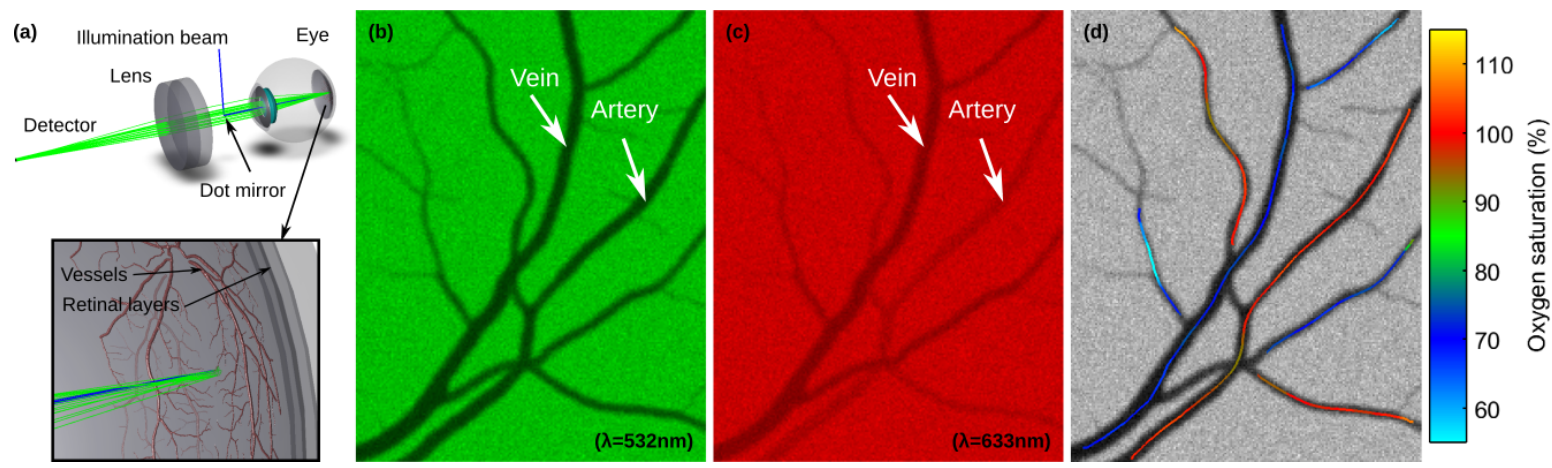

Fig. 1. Scanning Laser Ophthalmoscope. (a) Optical setup including eye with retinal vasculature. The illumination beam is scanned through the retina (scanning mechanism not shown) yielding images $(b, c)$. Two-wavelength oximetry is computed at tracked vessels (arteries and veins) in (d). 
This example shows an holistic modelling where rays are traced from source to detector: modelling light propagation in optical components and biological tissue enables to simulate instrument response to intricate interactions of light with complex structures.

The second example is the modelling of 3D imaging of a neuron cell using a Light-Sheet Fluorescence Microscope, Fig. 2. The optical setup, sketched in (a), comprises an illumination arm that forms a light sheet, and an imaging arm that records the excited fluorescence. The excitation rays are used to calculate the angular spectrum representation of the illumination beam, and the beam propagation method is used to calculate the diffracted illumination; additionally, scattering in the media (under the Born approximation) can be included through stochastic variations of the refractive index, results in (b-e). Recording the fluorescence signal from the sample's volume whilst scanned through the light sheet yields its 3D reconstruction, shown in (f).
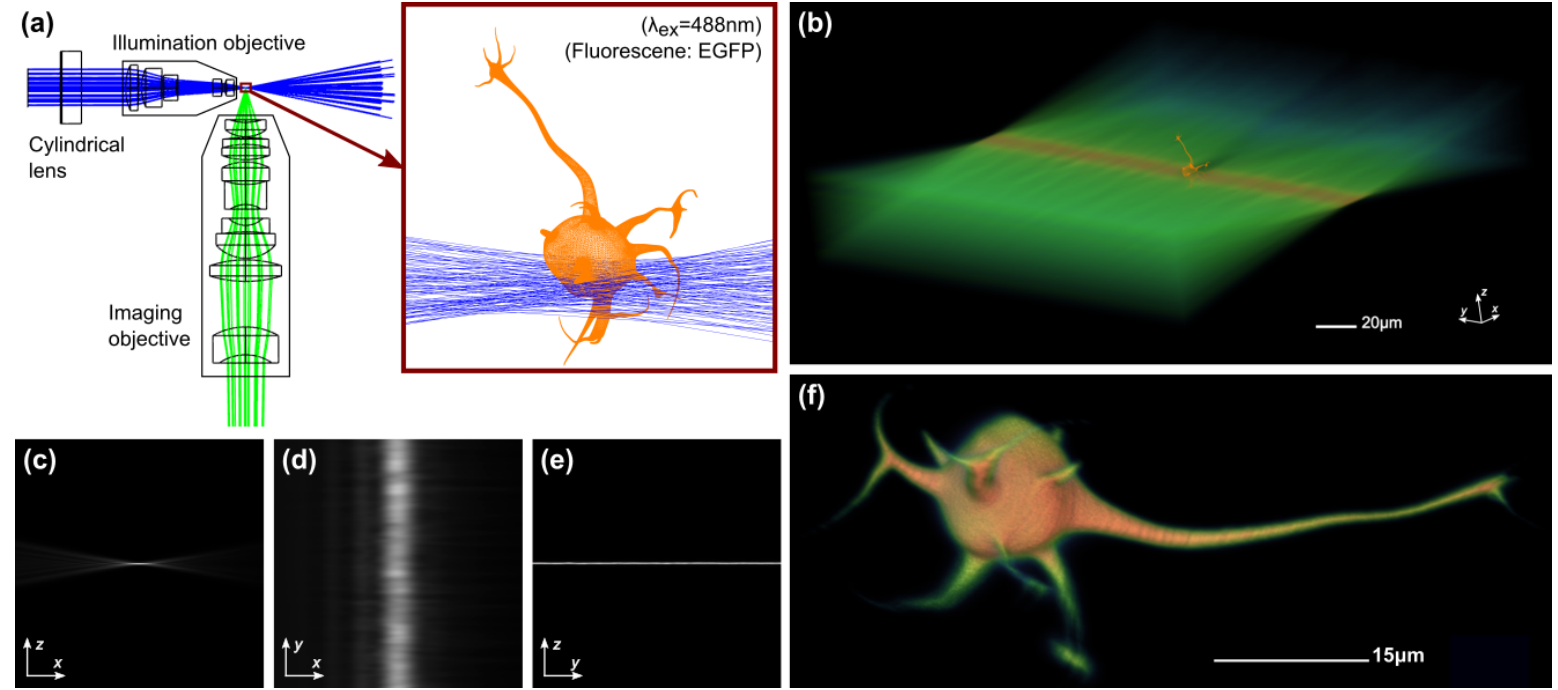

Fig. 2. Light-Sheet-Fluorescence Microscope. (a) Optical layout and sampling of the excitation beam, (b-e) calculated illumination beam, and (f) 3D reconstruction following plane scanning.

\section{Conclusions}

We have reported modelling showcases the potential of holistic modelling of imaging systems including turbid media through Monte-Carlo optical raytracing. We provide two add-on software packages for Zemax-OpticStudio ${ }^{\circledR}$ $[3,4]$ that enable such modelling. The first implements polarisation-sensitive Mie scattering tracking phase and polarisation [2]. The second is based in a three-step process in which: $(i)$ the angular spectrum of a beam is calculated, (ii) the beam propagation method is used to calculate the 3D diffractive intensity patterns, (iii) fluorescence rays are generated inside a sample volume according to the calculated diffracted excitation.

By exploiting the mature development of commercial optical raytrace software, integrating Monte-Carlo modelling of photon transport in tissue, these approaches offer the simulation of biological imaging systems, including realistic optical components (manufacturers' prescriptions may be readily used) and rigorous light-tissue interaction with arbitrary structures (available or modelled CAD models are readily imported, without need for writing computer code) to the expert and non-expert.

Such modelling is of increasing importance as enables $(i)$ investigating complex light/tissue interactions, joint optimisation of parameters including optical instrument and samples, construction of accurate inverse MonteCarlo models, generation of forward models for system inversion, and generation of realistic synthetic data for training of data-starved machine learning approaches.

\section{References}

1. V. Ntziachristos, "Going deeper than microscopy: the optical imaging frontier in biology," Nature Methods 7, 603-614 (2010).

2. G. Carles and A. R. Harvey, "Monte-Carlo Simulation of Imaging Systems with Turbid Media through Optical Ray-Trace," in Imaging and Applied Optics 2016, (Optical Society of America, 2016), paper MW2I.2.

3. G. Carles, "Ray Tracing Polarisation-sensitive Mie Scattering," Version v3, https://github.com/gcarles/RayPolMieScattering

4. G. Carles, "Diffracted beam propagation and fluorescence," https://github.com/gcarles/ZemaxDiffractionBeamFluorescence 